\title{
Application of PIV and PTV techniques to study propensity for thrombogenesis in PHV
}

\author{
M. Grigioni ${ }^{1}$, C. Daniele ${ }^{1}$, A. Balducci' ${ }^{1}$ D'Avenio', G. Querzoli ${ }^{2}$, \\ G.P. Romano ${ }^{3}$, Barbaro V.' \\ 'Laboratory of Biomedical Engineering, Istituto Superiore di Sanità, Rome, Italy \\ ${ }^{2}$ Departement of Engineering for the Territory, University of Cagliari, Cagliari, Italy \\ ${ }^{3}$ Department of Mechanics and Aeronautics, University of Rome "La Sapienza", \\ Rome, Italy
}

\begin{abstract}
The evaluation of the performances of a prosthetic heart valve (PHV) is related to the efficiency in replacing the diseased native valve. However, critical aspects remain in the post-implantation stage, due to the damage on red blood cells when the induced mechanical stresses overcome a certain threshold; furthermore, undesired effects of platelets adhesion and activation could take place due to high residence times.

The purpose of this study is to show how to obtain information about residence times downstream of a PHV by means of Particle Tracking Velocimetry (PTV), in virtue of the fact that there is a positive correlation between particle residence time and thrombogenesis. The in-vitro experiments were performed with $1 \mathrm{l} / \mathrm{min}$ mean cardiac output and $70 \mathrm{bpm}$ heart rate on a $19 \mathrm{~mm}$ aortic valve. The test fluid was a $33 \%$ water-glycerine solution with $50-150 \mu \mathrm{m}$ amberlite seeding particles. Characteristic adimensional numbers were $\operatorname{Re}=3214$ and $W_{0}=10.6$.

The Lagrangian approach allows following spatial and tidal evolution of the seeding particles, tracing their trajectories and calculating their velocities during each phase of the cardiac cycle. In this way it is possible to classify each region of the aortic root in terms of the associated average particle residence time; consequently, the potential for thrombogenesis can be evaluated quantitatively. The first relevant result consists of the evaluation of the maximum residence time that was found to be lower than cardiac cycle duration, although a low flow regime was used. Moreover, the capability of the two techniques to assess both shear stress distribution and related exposure times, allows the determination of critical regions and an estimation of the amount of blood subject to potential damage in relation to the total mass flow.
\end{abstract}




\section{Introduction}

\subsection{Background}

The assessment of blood damage, related to the implantation of mechanical heart valves, has become a very important issue in evaluating the performances of cardiovascular prostheses. Whereas technological improvements ensure an increasing durability and reliability of the implantable devices, blood traumatization always depends on the mechanical design of the device, on the presence of hinges and rotating parts and on the complexity of the generated flow field.

Particular attention has been placed on the estimation of the red blood cells (RBC) damage and on the platelets activation and deposition due the combination of stresses and residence times. Many authors have given estimations of the maximum stress acting on the blood in prosthetic heart valves (PHV) when systolic flow rate reaches the highest values, determining whether or not it has been exceeded the threshold value for $\mathrm{RBC}$ haemolysis $[1,2]$. Other works have focused their attention on the formulation of indices for the haemolysis estimation, due to cumulative stresses acting on blood particles in the operation of blood pumps or vascular access devices $[3,4]$. Those indices have been also applied on bioprosthetic heart valves to assess their tendency in damaging red blood cells by means of PIV technique [5]. Moreover, the tendency to platelet deposition has been evaluated on blood pumps and test protocols have been developed to improve the assessment of the causes of thrombogenesis [6].

Critical regions for thrombus formation in mechanical heart valves have been recognized to be the hinge areas of the leaflets, because of their low velocity and high shear rates during the opening phase. Valvular stenoticity, in association with the unsteady flow condition, could also promote the formation of recirculating regions downstream of the prosthesis, which may induce platelet activation. In addition, high values of the turbulent shear stress may damage the erythrocytes causing their membrane to release haemoglobin and other substances that may contribute to platelets aggregation.

\subsection{Integration of PIV and PTV}

The implementation of both Particle Image Velocimetry (PIV) and Particle Tracking Velocimetry (PTV) to the in-vitro testing of PHV is useful for the estimation of the potential damages because of the their different point of view. The Eulerian analysis, performed by PIV, gives information about the instantaneous structure of the flow field. That means the possibility to calculate flow variables such as velocity, vorticity and stresses in the whole test section, relative to the same instant. Otherwise the Lagrangian description, performed by PTV, follows the particles while they cross the test section, giving information about their position and residence time within each region. Thus, it is possible to 
correlate average residence times within the aortic root with average stresses acting on particle passing through the test section.

\section{Materials and methods}

\subsection{Experimental equipment}

The left heart pulse duplicator used was the one originally proposed by Professor Black of the Sheffield Royal Hallamshire Hospital and properly modified with the introduction of a blown-glass model of the aortic root [7].

The system was set to a heart rate of $70 \mathrm{bpm}$ and an imposed stroke volume of $1 \mathrm{l} / \mathrm{m}$ (Table 1), with a mean aortic pressure of $100 \mathrm{mmHg}$. Characteristic adimensional numbers, related to these setting parameters, were $\operatorname{Re}_{\mathrm{Ref}}=3214$ and $\mathrm{Wo}_{\mathrm{Ref}}=10.6$, calculated considering a reference velocity $\mathrm{U}_{\mathrm{Rer}}=0.626 \mathrm{~m} / \mathrm{s}$ (maximum instantaneous velocity averaged on flow meter section), $v=3.7 \mathrm{cSt}$ (blood cinematic viscosity) and $\mathrm{D}=19 \mathrm{~mm}$. Test fluid was a water - glycerine solution about $33 \%$ and seeding particles were made of a basic anion exchanger (50-150 $\mu \mathrm{m}$ diameter). The lighting and image acquisition system used for the experiments consists of an infrared diode laser with output power up to $12 \mathrm{~W}$ and wavelength $=800 \mathrm{~nm}$, an infrared sensitive camera, set to frame rate $=1 \mathrm{kHz}$, resolution $=320 \times 156$ and $1 / 1000 \mathrm{~s}$ shutter opening. An acquisition card was connected to a PC to download images from the video camera.

\subsection{PIV and PTV analysis}

Mean velocity fields were obtained by an averaging procedure applied to the PIV velocity fields having the same phase, selected among the recorded heart cycles. The number of cycles used to average velocity fields was 90 in the valve mid-plane. The latter comprises one sinus of Valsalva, intersected by the measurement plane at the right side of test section, while the other two sinuses are symmetrically disposed at opposite sides of the measurement plane. Each velocity field was calculated using commercial PIV analysis software (AEA Visiflow), setting the interrogation box (IB) to $32 \times 32$ pixels dimensions with about $70 \%$ overlap between consecutive boxes.

The Lagrangian trajectories were obtained by processing subsequent frames by means of an original method. The method proceeds by individuating centres of gravity of particles in each frame and then looking for centres referring to the same particle during tidal evolution. This is obtained using external information about maximum displacement and acceleration [8].

Residence times were subsequently estimated by dividing the test section in subdomains. Then the number of temporal samples in which each particle remains inside the same sub-domain was computed by following all the recorded trajectories passing through the aortic root during the phase of interest. The procedure is completed by normalizing the total calculated duration of residence by the number of trajectories followed by the particles, obtaining residence times in seconds. 
Table 1: Systolic flow rate

\begin{tabular}{|l|c|c|}
\hline Opening beginning & $135 \mathrm{~ms}$ & $11.6 \mathrm{ml}$ \\
\hline Systolic acceleration & $150 \mathrm{~ms}$ & $94.2 \mathrm{ml}$ \\
\hline Systolic peak & $165 \mathrm{~ms}$ & $196.9 \mathrm{ml}$ \\
\hline Systolic deceleration & $190 \mathrm{~ms}$ & $135.2 \mathrm{ml}$ \\
\hline Reduced ejection & $300 \mathrm{~ms}$ & 41.8 \\
\hline Closing beginning & $380 \mathrm{~ms}$ & $-11 \mathrm{ml}$ \\
\hline Valve closed & $428 \mathrm{~ms}$ & $-24 \mathrm{ml}$ \\
\hline
\end{tabular}

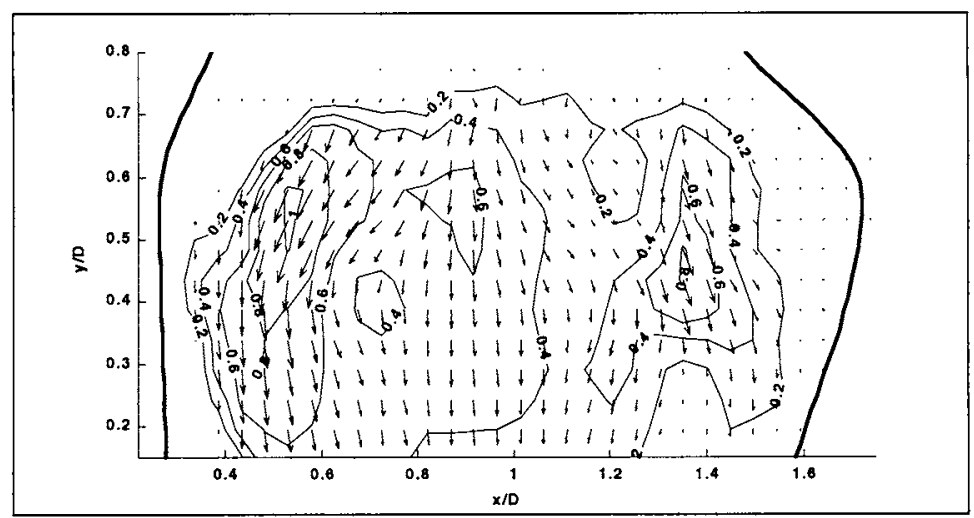

Figure 1: PIV mean velocity field at $\mathrm{t}=175 \mathrm{~ms}$ from cycle beginning

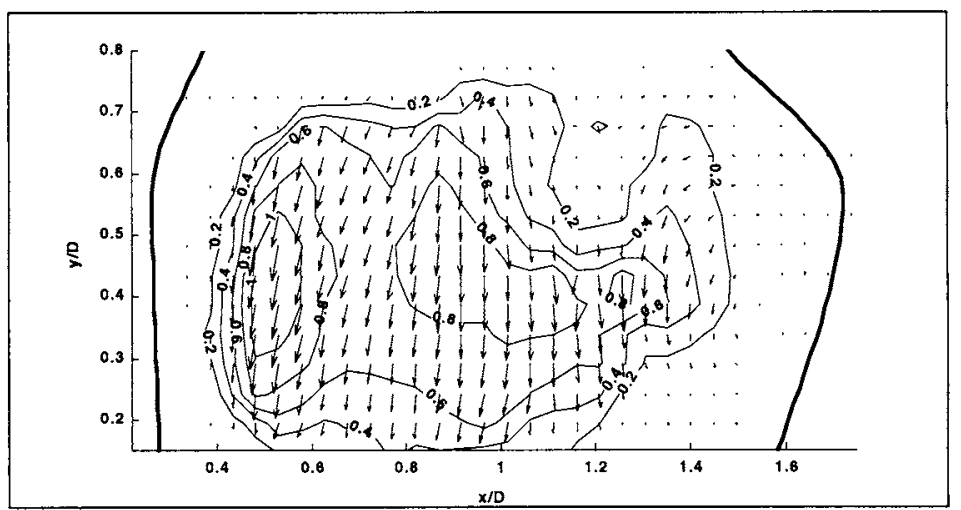

Figure 2: PIV mean velocity field at $\mathrm{t}=190 \mathrm{~ms}$ from cycle beginning 


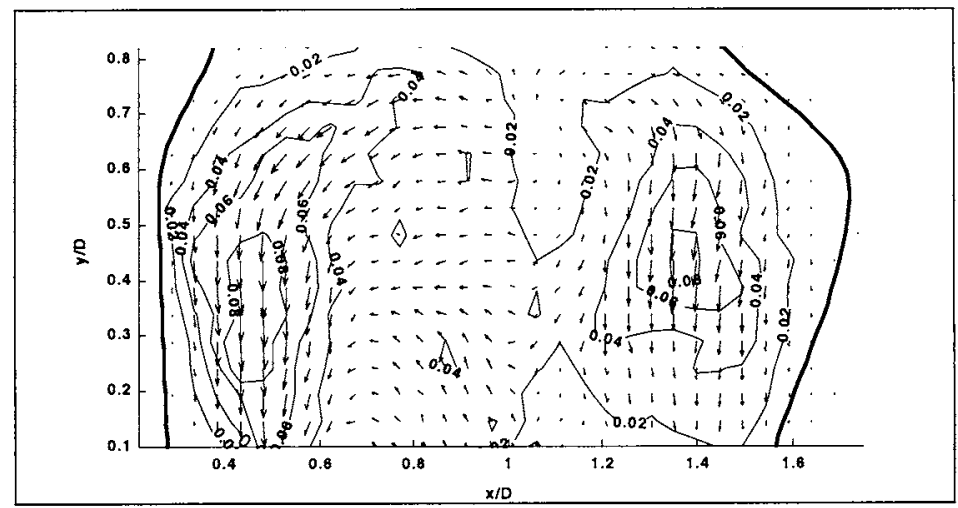

Figure 3: PIV mean velocity field at $\mathrm{t}=400 \mathrm{~ms}$ from cycle beginning

\section{Results}

\subsection{Description of the flow field}

Figures 1 and 2 show two PIV velocity fields referring, respectively, to systolic peak and to the first part of the the systolic deceleration. They have been chosen because of their capability in highlighting the instantaneous structure of the flow field during the fastest part of the systolic phase. The three jets configuration outgoing from the valve at systolic peak is clear from Figure 1, with particular attention to the vortical structure released downstream of the leaflets and in the sinus region. The effect of the deceleration increases the dimensions of the sinus fluid recirculation and squeezes the fluid toward the duct centre (Figure 2). In Fig. 3, the velocity field is reported for the end-systolic phase, at $400 \mathrm{~ms}$ after the cycle beginning.

\subsection{Residence times and Reynolds stresses}

The systolic residence time is presented in Figure 4: three zones are characterized by a higher value than that found in the rest of the section. The residence time relative to the lower left part of the aortic root is a consequence of the final part of the systolic phase (Figure 3), when the flow direction changes because of the valve regurgitation. It is located at the wall proximity while the associated shear intensity is low: this zone could be critical for potential thrombus formation due to platelets adhesion to the aortic wall. The other two zones of high residence time correspond to the right side of the section. They can be explained by the effect of the recirculation within the sinus and its interaction with the main flow. The mean systolic Reynolds stress also shows three main regions having higher values than the rest of the flow field. Figure 5 provides the systolic Reynolds stress contour map: it is interesting to remark the position of 


\section{6}

Simulations in Biomedicine $V$

the zones with the highest values set at the interface of the lateral jets with the central one and at the interface between the right jet and the recirculating region in the sinus. They are responsible for the possible stress induced damage to the membrane of the $\mathrm{RBC}$, due to their higher order of magnitude than that related to the viscous stresses.

\subsection{Haemolytic potential}

The knowledge of the Eulerian maps of Reynolds stress in the aortic root, during each temporal sample (1 ms) of the cardiac cycle, is the basis for the correlation with the position of the particle during its travel across the aortic root, obtained by the Lagrangian analysis. Figure 6 reports six selected trajectories that begin at the systolic peak and cover the whole test section. Across these trajectories the haemolytic potential was computed (equations (1) and (2)) by the formulation proposed by Giersiepien [9] and plotted in Figure 7:

$$
\begin{gathered}
\Delta I_{i}=a b\left(\tau_{x, i}^{d}\right)\left(t_{\mathrm{exp}, i}^{b-1}\right) \Delta t_{\mathrm{exp}} \\
I=\sum_{i=1}^{t_{\mathrm{exp}}} \Delta I_{i}
\end{gathered}
$$

where $\tau_{x y}$ and $\mathrm{t}_{\text {exp }}$ are expressed in $\mathrm{N} / \mathrm{m}^{2}$ and $\mathrm{s}$, respectively, while $a=3.62 \times 10^{-5}$, $b=0.785$ and $d=2.416$. The index is computed taking account of the tidal evolution of the particle trajectories across the aortic root. The time separation $\Delta t_{\text {exp }}$ between two successive detections of the centres of gravity of a particle is fixed to $1 \mathrm{~ms}$ by the image acquisition rate; $\tau_{x y, i}$ is the shear stress value acting during the $i$-th observation time interval $\Delta t_{\exp }$ and $t_{\exp , i}$ is the time spent by the blood particle to reach the $i$-th observation point (from the starting observation point).

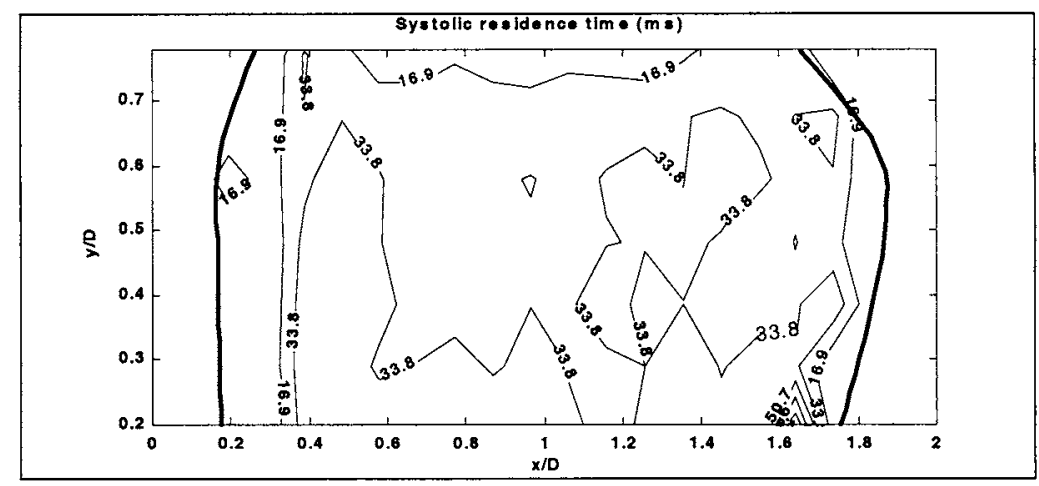

Figure 4: Mean systolic residence time in ms 


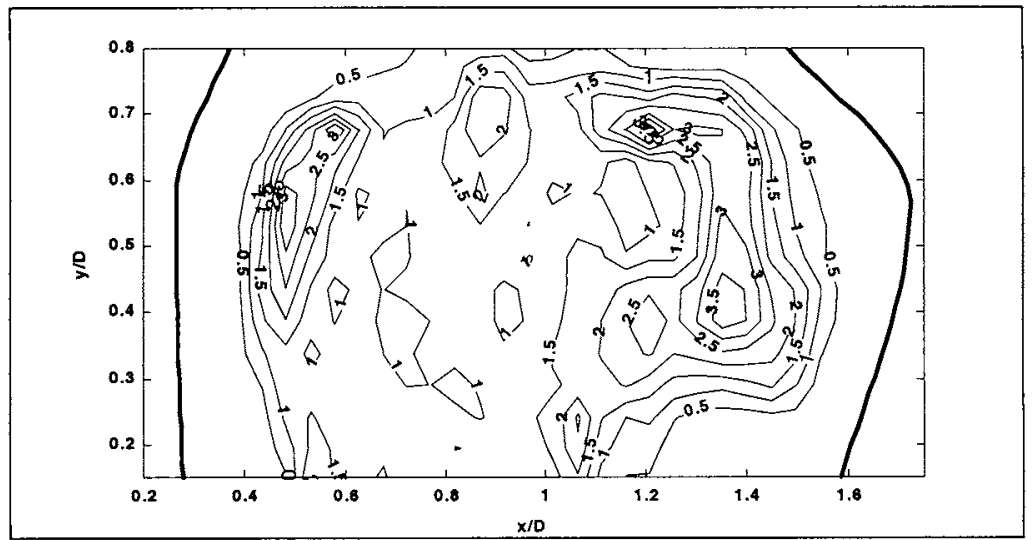

Figure 5: Mean systolic Reynolds stress in $\mathrm{N} / \mathrm{m}^{2}$

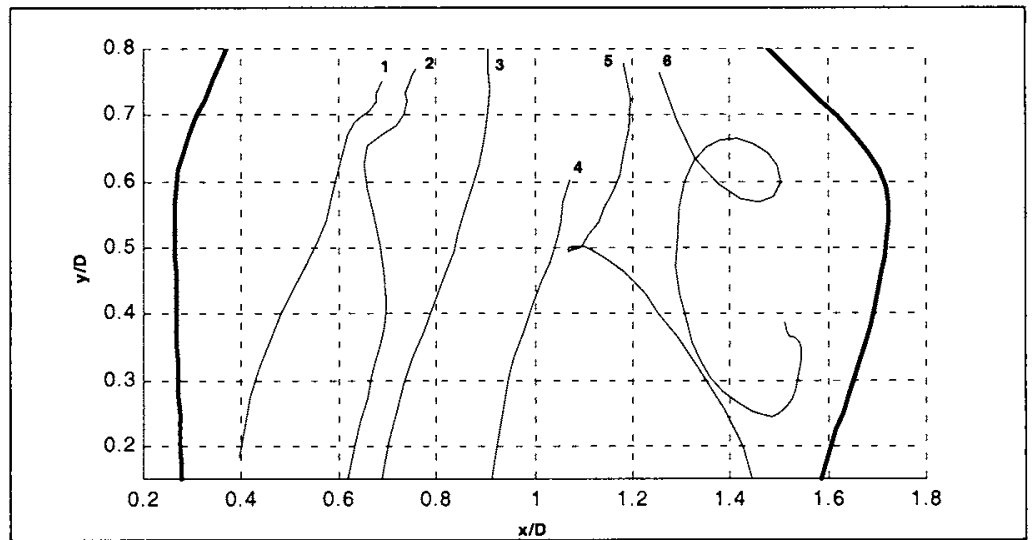

Figure 6: Selected trajectories

\section{Discussion}

The relevance in obtaining information about the loading on blood particles stems from the consequences acting on patiens with artificial implants. Particular care has to be placed on mechanical valve design; it is always necessary an anticoagulation treatment after the substitution of a natural valve with an artificial one of the mechanical type. It is necessary to improve the knowledge of the blood damage induced by the implant in order to tailor the pharmacological treatment of the implanted patients. This improvement may be attained by analyzing the characteristics of the flow field downstream of the valve. Recirculating regions are usually the first cause for the existence of zones characterized by high residence times. Furthermore, their interface zone with the main flow is also an important fluid dynamical feature, since it creates high 


\section{8}

Simulations in Biomedicine $V$

velocity gradients, responsible for the vortex stretching and for the extraction of turbulent energy, in association with high turbulent stresses.

The present study decribes the application of experimental laser techniques based on particle tracking to assess the possibility of the flow field downstream of a mechanical bileaflet heart valve to activate thromboembolic and haemolytic processes in blood particles. This analysis is made with the implicit assumption that blood particle do not deviate from the flow path of the plasma for a particle concentration up to to $50 \%$ (this is certainly not a limiting assumption, considering the average values of the haematocrit). A low flow regime was used $(1 \mathrm{l} / \mathrm{min}$ ) to measure particle residence times in the worst flow condition, but it did not avoid turbulent phenomena to be produced due to high Reynolds number and unsteady flow conditions $(\operatorname{Re} \approx 3200$ ). A recirculating region develops in the sinus of Valsalva, causing an increase of residence times during the ejection phase; moreover, the valvular regurgitation reverses the flow direction allowing the occurrence of another region of high residence time, near the wall in the region opposite to the sinus intersected by the measurement plane. The configuration with three orifices, characteristic of the bileaflet valve, creates three high shear regions that may be dangerous for haemolysis with physiological flow rates. The combination of the two effects, i.e., high residence time and stress-induced damage may produce a double effect on those particles which firstly pass through high shear region and subsequently get involved in fluid recirculation.

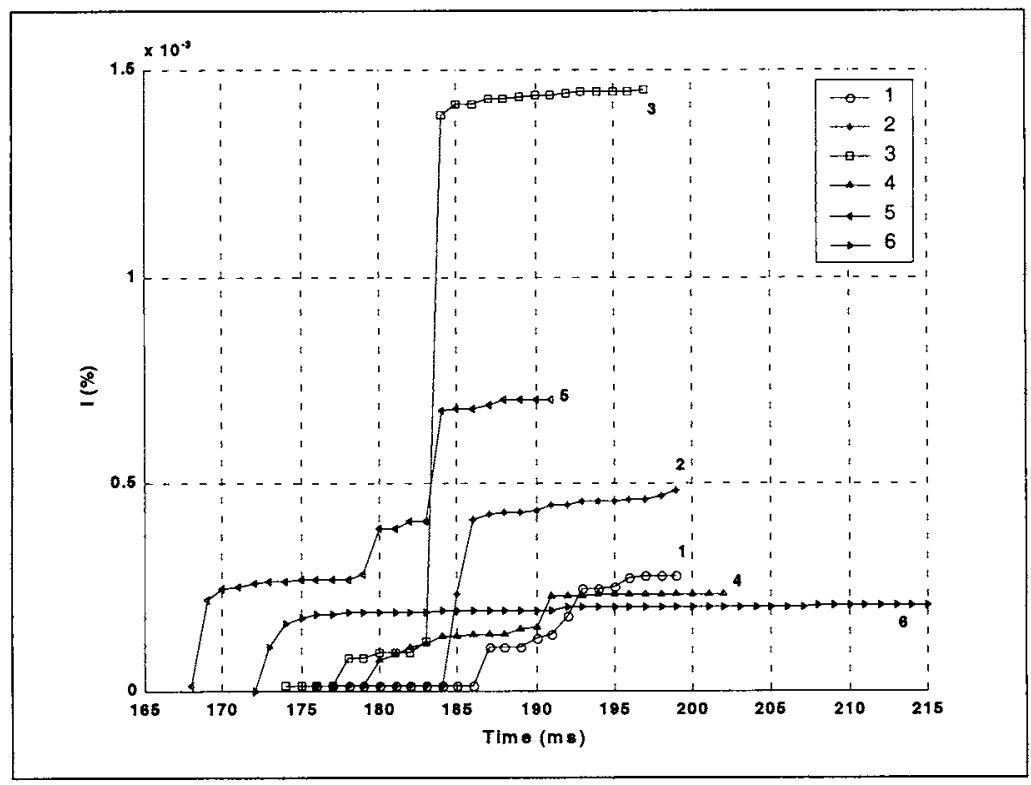

Figure 7: Haemolytic potential along particle trajectories 


\section{References}

[1] Grigioni M., Daniele C., D'Avenio G., Barbaro V. A discussion on the threshold limit for hemolysis related to Reynolds shear stress. J Biomech; 32(10), pp. 1107-12, 1999.

[2] Lu P.C., Lai H.C., Liu J.S. A reevaluation and discussion on the threshold limit for hemolysis in a turbulent shear flow. J Biomech, 34(10), pp. 1361-4, 2001.

[3] Apel J., Paul R., Klaus S., Siess T., Reul H. Assesment of hemolysis related quantities in a microaxial blood pump by computational fluid dynamics. Artif Organs, 25(5), pp. 341-347, 2001.

[4] Grigioni M., Daniele C., Morbiducci U., Di Benedetto G., D'Avenio G., Barbaro V. Mechanical blood trauma potential in vascular access devices: a comparison of case studies. Int J Artif Organs, 25(9), pp. , 2002.

[5] Lim W.L., Chew Y.T., Chew T.C., Low H.T. Pulsatile flow studies of a porcine bioprosthetic aortic valve in vitro: PIV measurements and shearinduced blood damage. J Biomech, 34, pp. 1417-1427, 2001.

[6] Paul R., Marseille O., Hintze E., Huber L., Schima H., Reul H., Rau G. In vitro thrombogenicity testing of artificial organs. Int J Artif Organs, 21(9), pp. 548-52, 1998.

[7] Barbaro V., Grigioni M., Daniele C., D'Avenio G. Principal stress analysis in LDA measurement of the flow field downstream of 19-mm Sorin Bicarbon heart valve. Technol Health Care, 6(4), pp. 259-70, 1998.

[8] Cenedese A., Querzoli G. Particle Tracking Velocimetry: measuring in the Lagrangian reference frame", in: Particle Image Velocimetry and associated techniques, Ed. M.L. Reithmuller, Lecture series 2000-01, Von Karmann Institute for Fluid Dinamics, Rhode de Saint Genèse, Belgium., 2000.

[9] Giersiepen M., Wurzinger L.J., Opitz R., Reul H. Estimation of shear stress related blood damage in heart valve protheses: in vitrocomparison of 25 aortic valves. Int $J$ Artif Organs, 13(5), pp. 300-306, 1990.

[10] Zimmer R., Steegers A., Paul R., Affeld K., Reul H. Velocities, shear stresses and blood damage potential of the leakage jets of the Medtronic Parallel bileaflet valve. Int J Artif Organs, 23, pp. 41-48, 2000.

[11] Schima H., Muller M.R., Tsangaris G., Gheiseder G., Schlusche C., Losert U., Thoma H., Wolner E. Mechanical blood traumatization by tubing and throttles in in vitro pumps test: experimental results and implications for haemolysis theory. Artif Organs, 17(3), pp. 164-170, 1993.

[12] Wootton D.M., Markou C.P., Hanson S.R., Ku D.N. A mechanistic model of acute platelets accumulation in thrombogenic stenoses. Ann Biomed Eng, 29(4), pp. 321-329, 2001.

[13] Bluestein D., Gutierrez C., Londono M., Schoephoerster R.T. Vortex shedding in steady flow through a model of an arterial stenosis and its relevance to mural platelet deposition. Ann Biomed Eng, 27(6), pp. 763$773,1999$. 
[14] Kunov M.J., Steinman D.A. Ethier C.R. Particle volumetric residence time calculation in arterial geometries. $J$ Biomech Eng, 118(2), pp. 158-164, 1996.

[15] Mallinger F., Drikakis D. laminar to turbulent transition in pulsatile flow through a stenosis. Biorheology, 39(3-4), pp. 437-441, 2002.

[16] Buestein D., Niu L., Schoephoerster R.T. Fluid mechanics of arterial stenosis: relationship to the development of mural thrombus. Ann Biomed Eng, 25(2), pp. 344-356, 1997. 